\title{
Silencing of TIM-3 Expression by miR-326 Affects Apoptosis and Proliferation of Human HL-60 Leukemia Cell Line
}

\author{
Maryam MOHAMMAD-GANJI ${ }^{1}$, Mazdak GANJALIKHANI-HAKEMI ${ }^{1}$, Vida HOMAYOUNI ${ }^{2}$, \\ Abbas REZAEI ${ }^{1}$, Hossein KHANAHMAD ${ }^{3}$ \\ ${ }^{1}$ Isfahan University of Medical Sciences, School of Medicine, Department of Immunology \\ ${ }^{2}$ Isfahan University of Medical Sciences, Acquired Immunodeficiency Research Center \\ ${ }^{3}$ Isfahan University of Medical Sciences, School of Medicine, Department of Genetics and Molecular Biology, \\ Isfahan, IRAN
}

\begin{abstract}
Leukemia Stem Cells (LSCS) are the main reason for drug-resistance and disease relapse in Acute Myeloid Leukemia (AML). Current drugs destroy normal Hematopoietic Stem Cells (HSCs) rather than LSCs. T cell immunoglobulin mucin-3 (TIM-3), an immune regulatory molecule, is a CD34+CD38- LSCs specific surface marker with high expression on these cells compared to HSCs. The interaction between TIM-3 and its ligand, Galectin-9 (Gal-9), mediates signaling pathways involved in apoptosis and proliferation. We hypothesized that miR-326 could have a suppressive activity on TIM-3 expression and hence, affects the proliferation and apoptosis processes in AML cells. Bioinformatics predictions were done using Mirwalk and Target Scan softwares. TIM-3 expression was induced on HL-60 cells by PMA. After miR-326 transfection, MTT, q-RT-PCR, flow- cytometery were performed to evaluate the cells survival and TIM-3 expression level. Then, after adding recombinant Galectin-9, apoptosis and proliferation rates were measured with Annexin- $V$ and CFSE assays, respectively. Flow cytometry assay confirmed our bioinformatics prediction of suppressive effect of miR326 on TIM-3 expression (66.4\% silencing) in HL-60 cell line ( $p=0.002)$. The qRT-PCR results were also confirmatory. Annexin- $V$ and MTT assays showed increased cell apoptosis and decreased cell survival, while data from CFSE assay indicated a severe reduction in HL-60 cells proliferation. Our results demonstrated that, miR-326 can silence TIM-3 expression in AML HL-60 cells. Moreover, it is shown that miR-326 can enhance AML cells apoptosis and reduce their proliferation and survival and hence, might be considered as a novel target for therapeutic approaches against AML.
\end{abstract}

Keywords: Acute myeloid leukemia, T cell immunoglobulin mucin-3, microRNA-326, Proliferation, Apoptosis

\section{INTRODUCTION}

Acute myeloid leukemia (AML) is a heterogeneous clonal disorder characterized by uncontrolled myeloid cell proliferation and block in myeloid differentiation that leads to their accumulation in the bone marrow and peripheral blood. ${ }^{1,2}$ Evidences demonstrate that AML is derived in a hierarchal system, driving from self-renewing leukemia stem cells (LSC). LSCs are able to continue to growth and proliferate leading to production of a large number of immature leukemia cells., LSCs are the main cause for the treatment failure and relapse of AML because LSCs are often resistant against most of the current cancer treatments such as irradiation and chemotherapy. ${ }^{4,5}$ Therefore, the LSC is the critical cellular target for a curative treatment of human AML. LSCs are represented in the CD34+CD38- fraction of AML cells. ${ }^{6-8}$ To root out the AML LSCs without killing normal HSCs, it is important to detect a molecule that expresses or functions specifically at the AML LSC stage. ${ }^{3}$ 
Recently, studies have shown CD123, CD44, CLL-1, CD25, CD32, CD96, and CD47 are preferentially expressed on surface cell of AML LSCs compared with normal HSCs. ${ }^{3,7,9}$ However, some of them are expressed in LSCs at a level insufficient to clearly distinguish, are represented also in normal HSCs, or are detected only in a fraction of AML cases. ${ }^{3,7}$

T cell immunoglobulin mucin-3 (TIM-3) is a type I glycoprotein which was first found in 2002 as a receptor on T-helper 1 (Th1) cells. ${ }^{10,11}$ Then, the expression of TIM-3 was discovered on other cells including DCs, monocyte/ macrophages, mast cells and other lymphocyte subsets. ${ }^{12,13}$ The S-type lectin galectin-9 (Gal-9) was then determined as a ligand for TIM-3. Gal-9 is a soluble molecule that is highly regulated by IFN- $\gamma$, and joins to oligosaccharides on the TIM-3 IgV domain. ${ }^{11}$ The TIM-3/ Gal-9 interaction is indicated to mediate both inhibitory and activating signaling pathways that have significant roles in infection, tumor immunity, inflammation, peripheral tolerance, and autoimmunity. ${ }^{10}$

Recent studies have indicated that TIM-3 is highly expressed as a surface molecule on CD34+CD38LSCs in most AML types. In contrast, TIM-3 is not existed in normal HSCs or lymphoid progenitor or myelo-erythroid population. ${ }^{14}$ Furthermore, recently, it has been demonstrated that TIM-3+ AML LSCs secrete galectin-9 in an autocrine manner and serum Gal-9 level is significantly increased in AML patients and in mice xenografted with primary human AML samples. It is also shown that signaling of TIM-3 by Gal-9 co-activates NF-kB and $\beta$-catenin pathways in LSCs that this signaling is commonly used for self-renewal of LSC and development of human AML. ${ }^{15}$ Therefore, TIM3 could be a promising candidate for eradication of AML LSCs ${ }^{3,7,8}$, through special suppressive tools like monoclonal antibodies or micro RNAs. ${ }^{14}$

MicroRNAs (miRNAs) are short single-stranded (19-25 nucleotides in length) non-coding RNAs. ${ }^{16,17}$ They can induce the degradation or suppression of the translation of the target mRNAs, usually by targeting of mRNA via 3'-untranslated regions (3'UTRs). ${ }^{18}$ MicroRNAs have important regulatory roles in cells including development, proliferation, differentiation and apoptosis of normal cells, also determination of the final phenotype of cancer cells. ${ }^{19}$ Base on their functions in cancers, miRNAs are divided into two groups. First group is called oncomiR (oncogene) that act as tumor developing by targeting and silencing tumor suppressor mRNAs. Second group is called tumor suppressor (tsmiR) that inhibits the expression of proto-oncogenes. ${ }^{19,20}$ Recent studies indicate that miRNAs could be useful for the detection, prevention and therapy of cancers. However, their application as biological markers is still in the initial stages. ${ }^{19}$

miR-326 located on chromosome 11q (http://www. mirbase.org). Dysregulation of miR-326 is associated with diseases and various physical process. ${ }^{21}$ Previously one study suggested that miR-326 plays an important role in stem cell regulation and cancer metabolism. ${ }^{22}$ Additionally, in another study, overexpression of miR-326 by transfection, resulted in decreased cell proliferation and elevated rate of apoptosis in CML CD34+ cells. ${ }^{23}$ Furthermore, context score percentile by bioinformatics predictions for silencing TIM-3 expression by miR-326 was predicted as $98 \%$ (http://www.targetscan.org). Therefore, we focused our studies on miR-326.

The human leukemia cell line, HL-60, is originated from a patient with acute promyelocytic leukemia. It was first diagnosed as FAB-M3 and afterward as FAB-M2 with an immature myeloid cells phenotype. ${ }^{14,24,25}$ The HL-60 cells are in promyelocytic, myeloblast and myelocyte stages. ${ }^{25,26}$ HL-60 cell line have also the capacity of expressing high level of TIM-3 following stimulation with PMA (phorbol myristate acetat). ${ }^{14,27}$ Therefore, this line could be considered as a useful model for study on AML. Based on high expression of TIM-3 on LSCs in AML and our bioinformatics predictions, we hypothesized that miR-326 could be a miRNA with suppressive activity on TIM-3 expression and may increase apoptosis and reduce proliferation rate in HL-60 cells. The results of this study may help to understand more aspects of TIM-3 roles in AML LSCs, also to new therapeutic approaches such as micro-RNA-based therapy for future. 


\section{MATERIALS AND METHODS}

\section{Bioinformatics Prediction}

Mirwalk 2.0 (http://zmf.umm.uni-heidelberg.de/ apps/zmf/mirwalk2/) was applied for Bioinformatics prediction of miRNA-TIM-3 interaction. In summary, in the "predicted targets module", "Gene-miRNA target" tab was chosen and the TIM-3 RefSeq ID (NM_032782.4) was put in the relevant box. Input parameters were edited on finding 3'-UTR and 10 databases were selected. Afterward, Target Scan 7.1 (http://www.targetscan.org/) was used to confirm Bioinformatics prediction of miRNA-TIM-3 interaction predicted by Mirwalk. Finally, the formal gene symbol of TIM-3 (HAVCR2) was put in the relative box and then, the query was submitted.

\section{Cell Culture}

HL-60 cell line (Pasture Inst, Iran) was maintained in Roswell Park Memorial Institute (RPMI) 1640 (Gibco, UK) supplemented with 10\% Fetal Buvin Serum (FBS; Gibco, UK) and 1\% antibiotics (100 $\mathrm{U} / \mathrm{ml}$ penicillin and $100 \mu \mathrm{g} / \mathrm{ml}$ streptomycin) (Sigma-Aldrich, USA). Cells were maintained at $37^{\circ} \mathrm{C}$ and in humid atmosphere, consisting of $5 \% \mathrm{CO}_{2}$.

\section{Cell Transfection}

The nucleotide sequence of miR-326 were acquired from http://www.mirbase.org as CCUCUGGGCCCUUCCUCCAG (accession number MIMAT0000756). About 24 hours before transfection, 105 HL-60 cells were placed in each well of 24 well plate containing $450 \mu 1$ RPMI 1640 per well without antibiotic or FBS. For overexpression of TIM-3, 50 ng PMA (Abcam, UK) were added to cells. Afterward, HL-60 cell transfection was performed with $50 \mathrm{nM}$ final concentration of has-miRNA-326 mimic (Qiagene, Germany) using the Xtreme GENE siRNA transfection reagent (Roche, Germany). $50 \mathrm{nM}$ final concentration of the LableIT siRNA delivery control conjugated with FITC (Mirus, USA) was used as both transfection indicator and scramble siRNA. Cells treated with only X-treme gene transfection reagent applied as mock control, and cells without treatment applied as negative control. After 4 hours incubation of the cells, the FBS and antibiotics were added to wells. Finally, after an overnight incubation, culture medium was exchanged and the plate were incubated for 24 hours. Evaluation of the transfection was performed by flow cytometry (BD Bioscience, USA).

\section{Cell Viability Test}

For assessing the viability of HL-60 cells, MTT (Methylthiazole terazolium) assay was applied. Briefly: $10 \mu \mathrm{l}$ of a $5 \mathrm{mg} / \mathrm{ml}$ MTT (Sigma-Aldrich, Germany) solution in PBS buffer was added to each well of 96-well plate (Orang, Belgium) containing $90 \mu \mathrm{l}$ of medium. After 1 hour incubation at $37^{\circ} \mathrm{C}$ and $5 \% \mathrm{CO} 2$, medium was removed and the cells were glaciated for 1 hour at $-80^{\circ} \mathrm{C}$ (New Brunswick scientific, USA). Then the purple formazan was liquated in $100 \mu \mathrm{l} /$ well dimethyl sulfoxide (DMSO) (Sigma-Aldrich, Germany) for 30 minutes while shaking. Optical density (OD) was measured by a mi $\neg$ croplate reader (M680 Bio rad, USA) at 590 $\mathrm{nm}$ (reference wavelength $630 \mathrm{~nm}$ ), and viability of the cells was defined as a percentage compared with un-transfected (negative) control cells.

\section{RNA Extraction, cDNA Synthesis and Quanti- tative RT-PCR}

Total RNA was extracted from HL-60 cell line through RNX Plus kit (Cinnagen, Inc. Iran). The cDNA was synthesized using the Revert Identify Transcription Kit (Fermentase, Germany). The resulting transcripts was applied for real time quantitative PCR on a Step One Plus ${ }^{\mathrm{TM}}$ real time DNA amplification system (Applied Biosystem, USA) with maxims SYBER Green qPCR master mix kit (Fermentase, Germany) and specific primers for each sample. Specific primers for TIM-3 and ACTB were determined by Allele ID 7.0 and their sequences are listed in Table 1. Thermal cycling was done using the following cycling conditions: $95^{\circ} \mathrm{C}$ for $10 \mathrm{~min}$ as the starting step, followed by 40 cycles of $95^{\circ} \mathrm{C}$ for $15 \mathrm{sec}, 60^{\circ}$ for $60 \mathrm{sec}$. The expression level of TIM-3 was obtained versus beta-actin (ACTB) expression as house-keeping gene. The relative expression was analyzed using $2-\Delta \Delta \mathrm{Ct}$ method. ${ }^{23}$ 


\begin{tabular}{|c|l|}
\hline \multicolumn{2}{|l|}{ Table 1. The sequences of specific primers of $\beta$-Actin and TIM-3 } \\
\hline Forward & 5-TTC GAG CAA GAG ATG GCC A-3 \\
\hline Reverse & 5-CAC AGG ACT CCA TGC CCA G-3 \\
\hline TIM-3 & Primer Sequence \\
\hline Forward & 5'- GTC ATC AAA CCA GCC AAG G-3' \\
\hline Reverse & 5'- CCC AGT GTC TGT GTC TCT-3' \\
& \\
\hline
\end{tabular}

\section{Flow Cytometry Analysis}

Flow cytometric analysis was done by FACS Callibuor instrument (Becton Dickinson Bioscience, San Jose, USA). Anti-human TIM-3-PE (eBiosciences, USA) was applied to measure the expression of TIM-3 protein on HL-60 cells. The results were analyzed using the Cell Quest Pro software (BD Bioscience, USA).

\section{Proliferation assay}

For assessing of the cells proliferation, carboxy fluorescein succinimidyl ester (CFSE; Biolegend, UK) was applied as instructed. Briefly: 1×106 HL-60 cells were cultured in RPMI medium, were stimulated with $50 \mathrm{ng}$ PMA. After 24 hours, miR326 was transfected using X-treme GENE siRNA into the HL-60 cells. Then $10 \%$ FBS and $1 \%$ antibiotics were added (4 hours later ) and after overnight incubation along with exchange of the cell culture medium, $2 \mu \mathrm{l}(1 \mu \mathrm{M})$ recombinant galectin-9 (Biolegend, UK) was added into each well and the well was maintained in Incubator for more 24 hours (cells without transfection were applied as controls, Gal-9 were added in both groups). On the last day, the HL-60 cells were stained with 10 $\mathrm{mM} \mathrm{CFSE} \mathrm{and} \mathrm{were} \mathrm{incubated} \mathrm{at} 37^{\circ} \mathrm{C}$ for $20 \mathrm{~min}$ in the dark. Then were washed 5 times with RPMI containing $10 \%$ FBS to remove excessive CFSE.
At the appropriate point in time, cells re-suspended in pre-warmed RPMI and incubated for $10 \mathrm{~min}$ utes. After incubation, cell proliferation were assessed immediately by FACS Callibuor instrument (BD Bioscience, USA).

\section{Apoptosis Assay}

For apoptosis assay, $1 \times 10^{6}$ cells were suspended in RPMI 1640 in 24 well plate. Cells were induced by 50 ng PMA, cell transfection was performed and the cells were incubated after addition of $2 \mu \mathrm{l}$ $(1 \mu \mathrm{M})$ recombinant Galectin-9 for 24 hours (untreated cells were applied as controls). Apoptosis was evaluated with an Annexin-V FITC Apoptosis Detection kit with PI (Biolegend; San Diego, California) according to the manufacturer's protocol. Annexin-V FITC was carried out to detect the phosphatidylserine on apoptotic cells. Propidium Iodide (PI) staining was used to discriminate necrotic cells. Apoptosis of the cells were detected by a FACS Callibuor flow cytometer (BD, USA).

\section{Statistical Analysis}

Statistical analysis was performed using SPSS 16.0 software (SPSS Inc, Chicago, USA). In order to compare among the groups, one-way ANOVA test and for compare results between patients and con- 
A
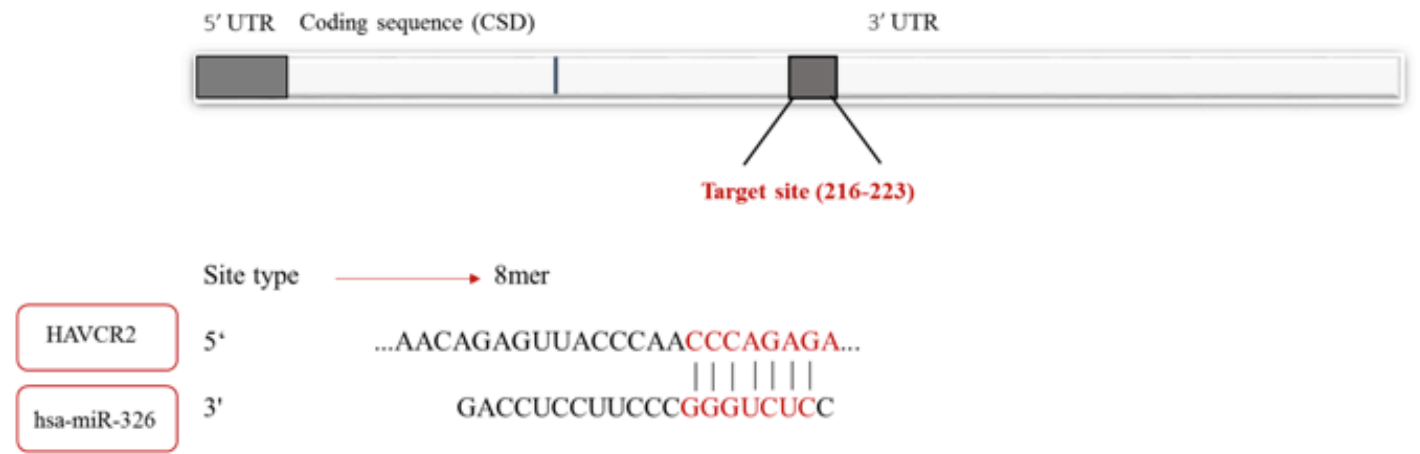

B

(216-223)

\begin{tabular}{|c|c|}
\hline Human & 5'--CC --UCUGUAUUUAAGCCAACAGAGUUACCCAAC-----CC-AGAGACUG---UU-AAUCA -- 3' \\
\hline Chimp & 5' --CC --UCUGUAUUUAAGCCAACAGAGUUACCCAAC-...-...-CC--AGAGACUG----UU-AAUCA -- 3' \\
\hline & 5'--CC --UCUGUAUUUAAACCAACAGAGUUACCCAAC--.--CC--AGAGACUG---UU-AAUCA -- 3' \\
\hline & 5' --CC--UCUGUAUCCCAGCUAACAGGGGUAUCCAAC-----CC--AGAGACUG---CC-AAUCA -- 3' \\
\hline & 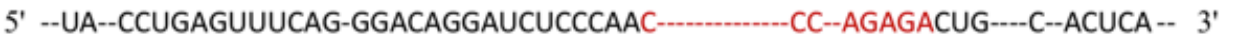 \\
\hline
\end{tabular}

Figure 1. Predicted hsa-miR-326 complementary sequence in HAVCR2 mRNA 3'-UTR. A) Describes the position of predicted MREs located in HAVCR2-3'UTR sequence and also shows pairing status of hsa-miR-326 and Forestalled MRE sequences. B) Displays conservation status of predicted complementary sequence in human and other mammals.

trol groups, independent student's t-test (Paired) were utilized. Results were assessed as means \pm relative standard deviation (Rel. SD) of at least three experiments. The $\mathrm{p}$ values of $<0.05$ are defined as significant.

\section{RESULTS}

\section{Bioinformatics Methods Predicted Silencing Ef- fect of miR-326 on TIM-3}

Using bioinformatics tools for predicting is a new method for challenging of prediction algorithms and for reducing experimental error and trials. ${ }^{28}$ In the Mirwalk output, inhibitory effect of miR326 on TIM-3 expression was predicted in six out of ten prediction algorithms including Microt4, miRanda, miRNAMap, RNA22, RNA hybrid and Targetscan software. These prophecy programs studied seed matching, energy, conservation, structure of miRNA and target positions interactions. These programs forestalled one miRNA recognition elements (MREs) within the HAVCR2-3'UTR sequence for hsa-miR-326 (Figure 1). "Validated targets module" indicated nothing for miR-326TIM-3 interaction which represented that there was no experimental study to validate this bioinformatics prediction. In the Target Scan output, context score percentile for silencing TIM-3 expression by miR-326 was predicted as $98 \%$ with an eight mer seed region and in Position 216-223 of 3' UTR of HAVCR2.

\section{PMA Increased TIM-3 Expression on HL-60 Cells}

The TIM-3 expression on HL-60 was induced with 50 ng PMA. The TIM-3 expression on HL-60 cell line was $76.48 \%$ after induction for 72 hours with PMA versus $0.08 \%$ without PMA treatment (Fig. 2).

miR-326 Decreased TIM-3 Expression on HL-60 Cell Line

In order to determine the inhibitory effect of miR326 on TIM-3, has-miR-326 mimic transfected 


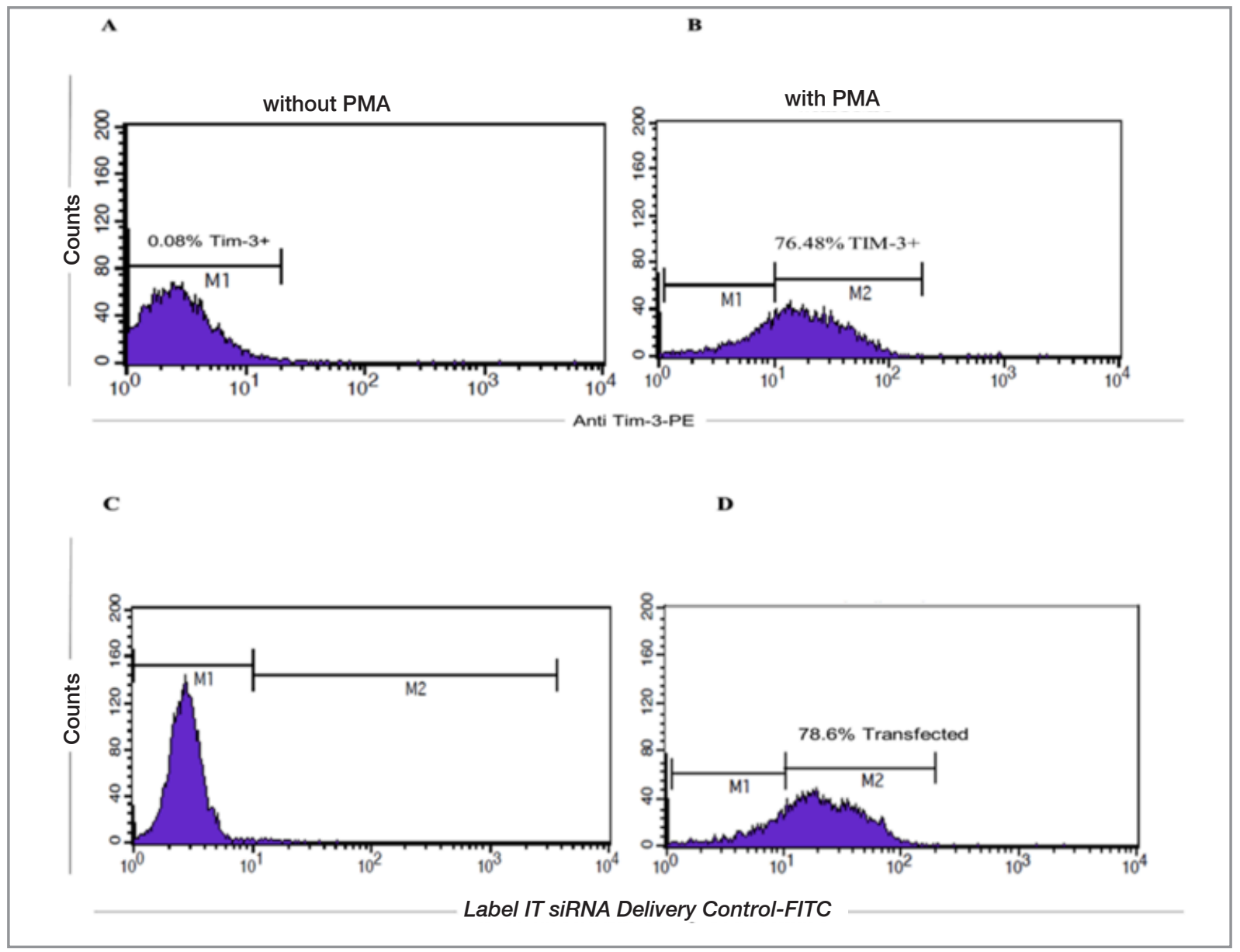

Figure 2. Analysis of TIM-3 expression on HL-60 cell line by Flow cytometry using anti TIM-3-PE antibody. Histogram analysis of Flow cytometry of negative control without PMA (A) in compare with HL-60 cells with PMA (B), indicates that TIM-3 expression on HL-60 cells was $76.48 \%$ after treatment with PMA for 72 hours. Also, analysis of transfection efficiency by flow cytometric analysis of HL-60 cells Histogram graphs of negative control (C), and HL-60 cells after transfection by Label IT® siRNA Delivery Control-FITC (D), show transfection efficiency was $78.63 \%$ into the HL-60 cells.

into HL-60 cells after 24 hours of treatment with PMA. Successful transfection of $78.63 \%$ of the cells were detected by flow cytometry method and using anti TIM-3-PE antibody (Figure 2). In transfected test group, flow cytometry analysis showed that only $33.6 \pm 6.9 \%$ of transfected cells expressed TIM-3 protein, as compared with un-transfected control $(100 \%)$ group, which indicates more than $66.4 \%$ gene silencing $(\mathrm{p}=0.002)$ (Figure $3 \mathrm{~A})$. As shown in Figure 2, there was no significant difference between negative, mock and scramble controls for TIM-3 expression. This data confirms that the silencing was specific.

The expression level of TIM-3 was also assessed in transfected and un-transfected HL-60 cells by quantitative RT-PCR. As it is obvious in figure 3B,
TIM3 mRNA level was $65.8 \pm 1.4 \%$ in compare with negative control $(\mathrm{p}=0.001)$. These observations showed $34.2 \%$ decrease in TIM-3 transcript level. As expected, there was no significant difference for TIM-3 transcript level between untreated, mock and scramble controls (Figure 3B).

\section{Mir-326 Decreased Proliferation of HL-60 Cells Stimulated with Gal9}

To approve whether miR-326 mediated TIM-3 suppression affects HL-60 cells proliferation, recombinant galectin-9 were added to the cells 24 hours after transfection. On the last day, the HL-60 cells were stained with CFSE. Then cell proliferation were performed immediately by FACS Callibuor instrument (BD Bioscience, USA). Interestingly, 


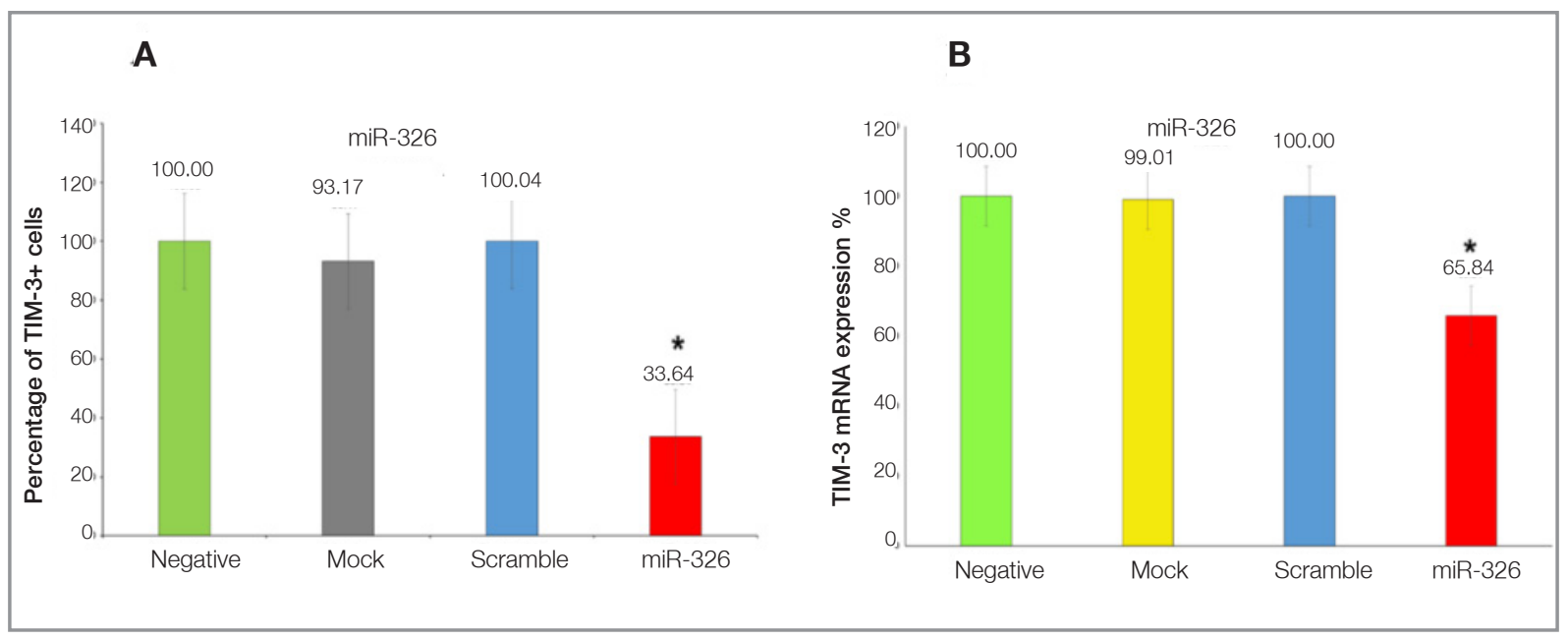

Figure 3. Analysis of the TIM-3 expression in HL-60 cells by flow cytometry 72 hours after transfection with miR-326 is displayed in the graph (A). The graph shows expression of TIM-3 on transfected cells was significantly suppressed in compare with controls ( $p<$ 0.05). TIM-3 expression level had no significant difference among negative, mock and scramble controls. Assessment of the TIM-3 expression in HL-60 cells by real time PCR 72 hours after transfection with miR-326 is displayed in the graph (B). TIM-3 mRNA was decreased in transfected cells compared to negative control $(\mathrm{P}<0.05)$. TIM-3 expression had no significant difference among negative, mock and scramble controls. Data are expressed as mean \pm Rel.SD of three independent experiments. Asterisk $\left({ }^{\star}\right)$ shows statistical significance.

decrease in TIM-3 expression was associated with $70.34 \%$ decrease in cell proliferation rate in transfected cells compared to un-transfected cells with miR-326 (Figure 4).

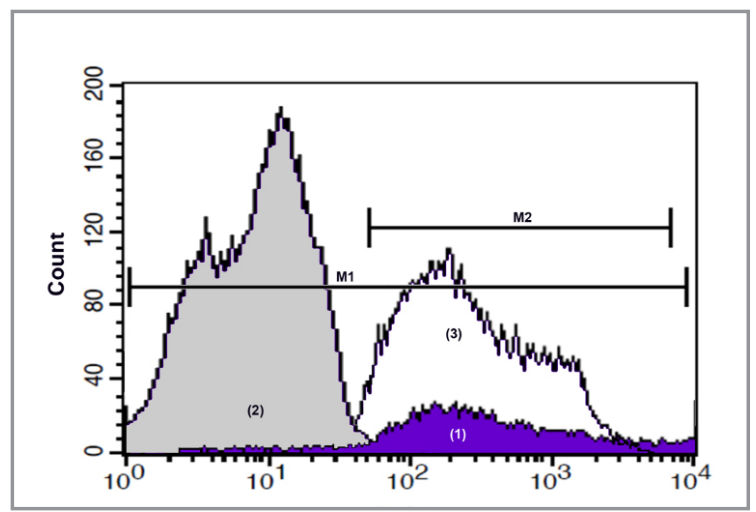

Figure 4. HL-60 cells proliferation was determined by flow cytometry $72 \mathrm{~h}$ after transfection with miR-326. Galectin-9 were added to cells $24 \mathrm{~h}$ after transfection, then cells were stained with CFSE in the last day. In the histograms, (1) shows untreated HL-60 cells proliferation; (2) shows HL-60 cells proliferation treated with Galectin-9 only; and (3) shows proliferation inhibition HL-60 cells treated with Galectin-9 and miR-326.As shown, TIM-3 suppression was associated with $70.34 \%$ inhibition of proliferation after transfection with miR-326.

\section{Inhibition of TIM-3 Decreased Cell Viability and Enhances Apoptosis in HL-60 Cells}

To assess the effects of TIM- 3 inhibition on HL60 cell viability, the MTT assay was applied after transfection with miR-326. Cell viability had not any significant difference between transfected cells in untreated, mock and scramble controls ( $p>0.05$ ). Whereas, viability of HL-60 cells transfected with the miR-326 was dramatically lower compared to the control groups $(\mathrm{p}=0.0001)$. The viability of the miR-326 transfected cells was $34.6 \pm 7.0 \%$ at 72 hours after transfection (Figure 5A). Therefore, the miR-326 by TIM-3 silencing generally decreases viability of HL-60 cells.

To investigate the possibility of apoptosis and necrosis after miR-326 overexpression and TIM-3 inhibition, we encounter the cells with Gal-9 and then, the cells were stained by Annexin-V FITC and PI 72 hours after transfection. Flow cytometry analysis of the transfeced cells stained with Annexin V-FITC and PI showed that TIM-3 inhibition by miR-326 enhanced cell apoptosis as compared to control (Figure 5B and 5C). Results showed that by $72 \mathrm{~h}$ after miR-326 overexpression up to $29 \%$ of the cells had been undergone apoptosis (early and 


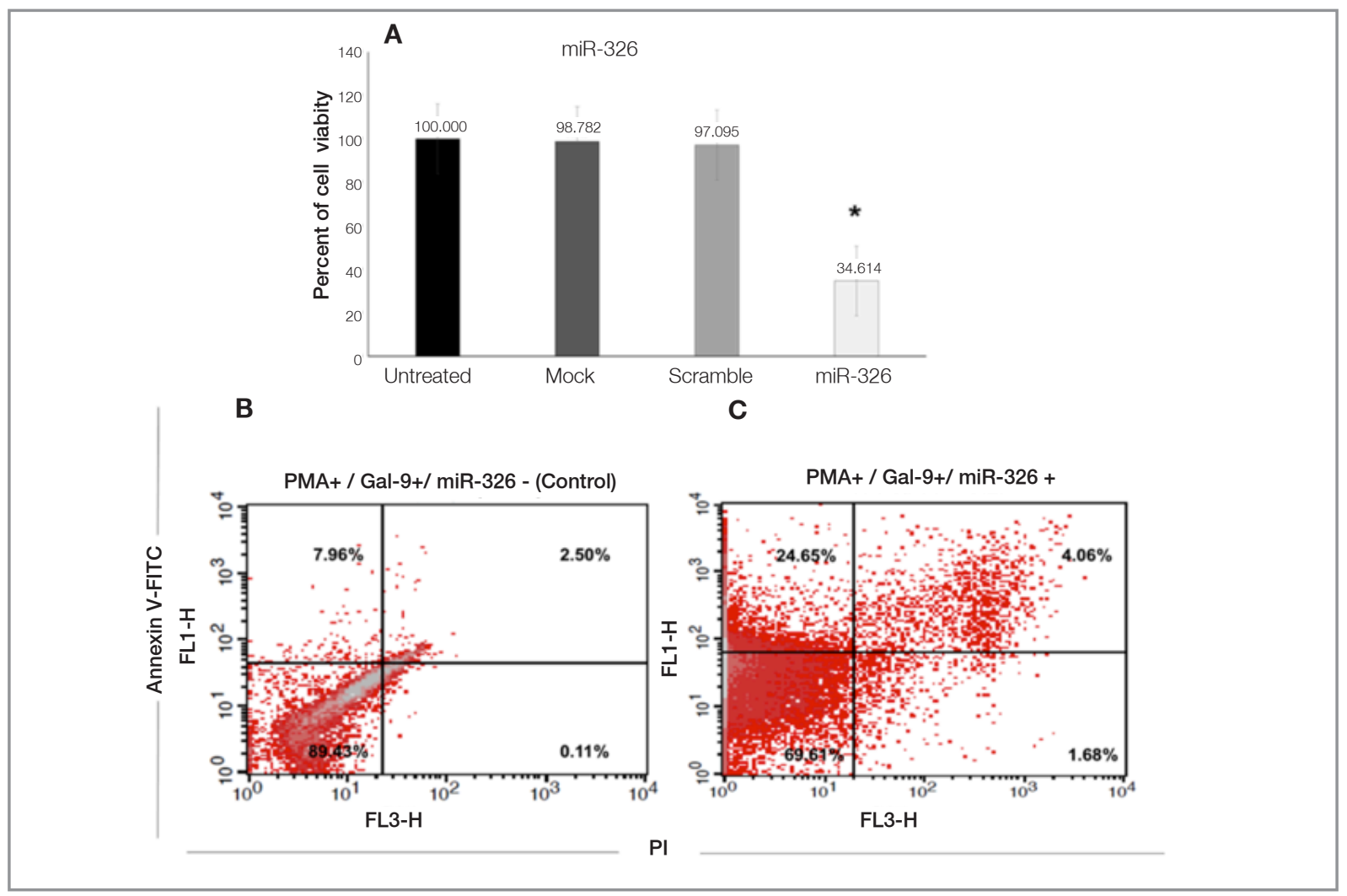

Figure 5. Assessment of Cell viability by MTT assay 72 hours after transfection with miR-326. As displayed in the graph (A), the cell viability in transfected $\mathrm{HL}-60$ cells is about $35 \%$ which is significantly lower than untreated cells. Conversely, there is no punctual difference among untreated, mock and scramble controls for cell viability. Results show that the miR-326 by TIM-3 gene silencing generally decreases cell viability of HL-60 cells. Data are expressed as mean \pm Rel.SD of three independent experiments. Asterisk $\left(^{*}\right)$ shows statistical significance. Analysis of miR-326 transfected HL-60 cells with Annexin V-FITC and PI. HL-60 cells after encountering with Gal-9 and 72 hours after transfection were stained by Annexin-V FITC and PI and were analyzed with flow cytometry. Dead cells were described as necrotic (PI-positive/ Annexin V-negative) or apoptotic (Annexin V-positive/PI-negative and Annexin V-positive/ PI-positive). Annexin $\vee$ histogram of negative control indicates that early and late apoptotic cells were $7.96 \%$ and $2.50 \%$, respectively (B). In contrast, Annexin $V$ histogram of HL-60 cells after 72 hours of miR-326 transfection display that the early and late apoptotic cells were up to $24.65 \%$ and $4.06 \%$, respectively (C).

late stages of apoptosis) (Figure 5C), compared to negative control with $10.46 \%$ apoptosis (early and late stages of apoptosis) (Figure 5B). As well as the ratio of necrotic cells in the miR-326 positive group with $5.74 \%$ (Figure 5C) was higher than control group with $2.61 \%$ (Figure 5B).

\section{DISCUSSION}

Acute myeloid leukemia is a serious and often a lethal cancer in which immature myeloid cells have rapid proliferation in the bone marrow associated with dysfunctional hematopoiesis. Recently, attention to cancer stem cells has been increased. There are strong documents that LSCs are subpopulation from AML that are resistant to immunotherapy and chemotherapy. Therefore LSCs are main reason for the treatment failure and reversion of $\mathrm{AML}^{23}$, and novel impressive leukemia therapeutics are necessary and required. Recent studies clearly suggest that TIM-3 is a surface marker which specifically expressed on CD34+CD38- AML LSCs fraction at a high level, while is not expressed on normal HSCs or other progenitors. ${ }^{3,9}$ One study indicated that anti-TIM-3 monoclonal antibody, ATIK2a, could root out human AML LSCs in immunedeficient mice injected with human AML cells without affecting normal hematopoiesis. ${ }^{3}$ High expression of TIM-3 in cancers like AML suggests that its expression might be responsible for LSCs survival. In one study, Kikushige et al added Gal-9 and HMG$\mathrm{BI}$ (High mobility group box 1 protein) to AML 
TIM-3 positive cells and found low expression of pro-apoptotic gene, BAX. ${ }^{29}$ It has been reported that interaction between microRNAs and their target genes involves in differentiation growth, apoptosis, growth and cell proliferation. ${ }^{30}$ Considering this findings and miRNA inhibitory function, we planned to study the effect of miR-326 on TIM-3 expression on an AML cell line, HL-60 and investigate the probable effect of TIM-3 on proliferation and apoptosis in leukemic cells using miRNA mediated gene silencing.

As we had predicted by bioinformatics tools, our experimental finding showed that miR-326 was able to significantly inhibit TIM-3 protein expression on HL-60 cells. Although TIM-3 mRNA level was reduced to $65 \%$, the difference with control was still significant. This could tell us that the mechanism of miR-326 action on TIM-3 expression was rather translation hindrance. In a recent study, Emamdoos et al. have shown that miR-125a$3 p$ prevents the expression of TIM-3 protein level more than $98 \%$. Their results of qRT-PCR method have demonstrated about $50 \%$ decrease in TIM-3 mRNA level. ${ }^{27}$ In another study, Fooladinezhad et al. have studied effect of miR-330-5p on TIM3 expression in HL-60 cells. They showed that miR-330-5p was able to suppress the expression of TIM3 protein with more than $98 \%$ in vitro. ${ }^{14}$ These data was in line with our findings about the effect of miR-326 on TIM-3 protein expression.

In the current study, using CFSE proliferation assay, we showed that decreased level of TIM-3 by miR-326, was associated with more than $70 \%$ decrease in cell proliferation rate. Hence, the impact of TIM-3 on AML cells proliferation was confirmed. According to our survey in the available data in different databases, our finding is the first one which shows that the suppression of TIM-3 expression in HL-60 cells by miR-326 can decrease their proliferation rate in vitro. The only comparable study performed by Babashah et al. has demonstrated that overexpression of miR-326 inhibits cell proliferation in primary CML CD34 cells. ${ }^{23}$ Wang et al. indicated that Up-regulation of miR-326 in lung cancer, decreased Phox2a and decreased cell proliferation in both A549 and H838 cells. ${ }^{21}$ This is in line with our results.
Apoptosis, or programmed cell death, play a key role in the control of the development and homeostasis of the hematopoietic system and deregulation of apoptosis disrupts balance between cell proliferation, cell survival and cell death and have an important role in the development of cancers, particularly leukemias. Moreover, tumors that have abnormalities in proteins involved in apoptosis are resistant to chemotherapy. Studies indicate that regularly there are abnormalities in one or more apoptotic pathways in leukemia cells that lead to survival advantage of these cells over their normal counterpart. ${ }^{31}$ It has been demonstrated that high level of anti-apoptotic Bcl-2 protein prevent apoptosis and is associated with enhanced number and repopulation potential of stem cells..$^{23}$ Additionally, it has been reported that in AML, overexpression Bcl-2 levels lead to resistance to chemotherapy, reduced rates of complete remission, and shortened survival. ${ }^{32}$

Another finding of ours in the present work was that the silencing TIM-3 using miR-326 decreased the viability of HL- 60 cells to $35 \%$ and enhanced the apoptosis of this cell line (in presence of Gal9) to about $30 \%$. Therefore, we confirmed that TIM3 is an important factor which promotes cell viability in AML cells. In one study, Kikushige et al. have indicated that after adding Gal-9 and HMGBI (High mobility group box 1 protein) to AML TIM$3+$ cells the level of pro-apoptotic gene, BAX, decreased. ${ }^{29}$ Wang et al. reported that miR-326 was down-regulated in lung cancer. They founded that enforced expression of miR-326, decreased Phox $2 \mathrm{a}$ and regulated cell apoptosis and metastasis in both A549 and H838 cells and inhibits tumor growth in nude mice. ${ }^{21}$ Karsy et al. indicated Glioblastoma Multiforme tumor-derived CSCs (cancer stem cells) were transfected with miR-326 and demonstrated reduced invasiveness, viability and clonogenicity as well as enhanced apoptosis. ${ }^{22}$ In another study, Babashah et al. have reported that overexpression of miR-326 by transfection downregulated the smoothened (Smo) signal transducer of Hedgehog (Hh) developmental signaling pathway, led to increased rate of apoptosis in CML CD34+ cells. $^{23}$ In another study, Lee J,et al. demonstrated GBM tumor-derived CSCs were transfected with miR-326 and showed reduced 
invasiveness, viability, and clonogenicity as well as inhanced apoptosis in vivo. ${ }^{33}$ Burak Uz, et al have shown that renin-angiotensin system (RAS) activity is elevated in AML and RAS inhibitors decreased the growth and colony-forming ability of AML cells in a dose-dependent manner. ${ }^{26}$ As we also predicted by Target Scan that miR-326 is able to suppress ACE expression, the anti-proliferative and pro-apoptotic effect of miR-326 showed in our study may confirm its role as a RAS inhibitor. However, this needs to be specifically confirmed.

These results are consistent with our findings about the importance of TIM-3 in survival of malignant cells and increase of apoptosis and miR-326 as a tumor-suppressor miRNA. Our results suggests suppressive effect of miR-326 on TIM3 expression on AML HL-60 cell line. However, the direct effect of miR-326 on TIM-3 should be confirmed in another study.

In Conclusion, since TIM-3 is a specific surface marker of LSCs in almost all types of AML with high expression and because of TIM-3 anti-tumor responses, we suggest that TIM- 3 could be considered as a helpful marker for discriminating LSCs from HSCs and inhibition of TIM-3 could be a hopeful strategy for eradication of AML LSCs. Furthermore, miR-326 can be considered as a critical target for future studies on AML and more investigations on the manipulation of expression levels of miR-326 could be useful in AML in a research setting.

\section{Acknowledgment:}

This research was financially supported by Isfahan University of Medical Sciences (Iran) with the grant number of 394421.

\section{REFERENCES}

1. Grove CS, Vassiliou GS. Acute myeloid leukaemia: a paradigm for the clonal evolution of cancer? Dis Model Mech 7: 941-951, 2014.

2. Saultz JN, Garzon R. Acute Myeloid Leukemia: A Concise Review. J Clin Med 5, pii: E33, 2016.

3. Kikushige Y, Shima T, Takayanagi S-i, et al. TIM-3 is a promising target to selectively kill acute myeloid leukemia stem cells. Cell Stem Cell 7: 708-717, 2010.
4. Zhou J, Chng W-J. Identification and targeting leukemia stem cells: The path to the cure for acute myeloid leukemia. World J Stem Cells 6: 473-484, 2014.

5. Riether C, Schürch C, Ochsenbein A. Regulation of hematopoietic and leukemic stem cells by the immune system. Cell Death Differ 22: 187-198, 2015.

6. Xiang-Xin Li X-PH, Chun-Hong Ma, Lu-Qun Wang. Tim-3 as a leukemia stem cell specific marker expressing on bone marrow mononuclear cells is a factor for prognosis evaluation in patients with acute myelogenous leukemia. Int J Clin Exp Med 9: 10979-10986, 2016.

7. Gao L, Yu S, Zhang X. Hypothesis: Tim-3/galectin-9, a new pathway for leukemia stem cells survival by promoting expansion of myeloid-derived suppressor cells and differentiating into tumor-associated macrophages. Cell Biochem Biophys 70: 273-277, 2014

8. Akashi K. TIM-3 Is a Novel Therapeutic Target for Eradicating Acute Myelogenous Leukemia Stem Cells. In: Innovative Medicine. Nakao K, Minato N, Uemoto Sh. Springer, 2015: 307-315.

9. Jan M, Chao MP, Cha AC, et al. Prospective separation of normal and leukemic stem cells based on differential expression of TIM3, a human acute myeloid leukemia stem cell marker. Proc Natl Acad Sci 108: 5009-5014, 2011.

10. Gleason MK, Lenvik TR, McCullar V, et al. Tim-3 is an inducible human natural killer cell receptor that enhances interferon gamma production in response to galectin-9. Blood 119: 3064-3072, 2012.

11. Sakuishi K, Jayaraman P, Behar SM, et al. Emerging Tim-3 functions in antimicrobial and tumor immunity. Trends Immunol 32: 345-349, 2011

12. Ngiow SF, Teng MW, Smyth MJ. Prospects for TIM3-targeted antitumor immunotherapy. Cancer Res 71: 6567-6571, 2011.

13. Anderson AC. Editorial: Tim-3 puts on the brakes. J Leukoc Biol 91: 183-185, 2012.

14. Fooladinezhad $\mathrm{H}$, Khanahmad $\mathrm{H}$, Ganjalikhani-Hakemi M, et al. Negative Regulation of TIM-3 Expression in AML Cell Line (HL-60) Using Mir-330-5p. Br J Biomed Sci 73: 129-133, 2016.

15. Kikushige Y, Miyamoto T, Yuda J, et al. A TIM-3/Gal-9 autocrine stimulatory loop drives self-renewal of human myeloid leukemia stem cells and leukemic progression. Cell Stem Cell 17: 341-352, 2015.

16. Braicu C, Tomuleasa C, Monroig P, et al. Exosomes as divine messengers: are they the Hermes of modern molecular oncology\&quest. Cell Death Differ 22: 34-45, 2015.

17. Xiong $\mathrm{Q}$, Yang $\mathrm{Y}$, Wang $\mathrm{H}$, et al. Characterization of miRNomes in acute and chronic myeloid leukemia cell lines. Genomics Proteomics Bioinformatics 12: 79-91, 2014. 
18. Huang $\mathrm{K}$, Dong $\mathrm{B}$, Wang $\mathrm{Y}$, et al. MicroRNA-519 enhances HL60 human acute myeloid leukemia cell line proliferation by reducing the expression level of RNA-binding protein human antigen R. Mol Med Rep 12: 7830-7836, 2015.

19. Garajová I, Le Large TY, Frampton AE, et al. Molecular mechanisms underlying the role of microRNAs in the chemoresistance of pancreatic cancer. BioMed Res Int 2014; 2014: 678401. doi: 10.1155/2014/678401.

20. Takahashi R-u, Miyazaki H, Ochiya T. The role of microRNAs in the regulation of cancer stem cells. Front Genet 4: e295, 2013. doi: 10.3389/fgene.2013.00295.

21. Wang R, Chen X, Xu T, et al. MiR-326 regulates cell proliferation and migration in lung cancer by targeting phox2a and is regulated by HOTAIR. Am J Cancer Res 6: 173-186, 2016.

22. Karsy M, Arslan E, Moy F. Current progress on understanding microRNAs in glioblastoma multiforme. Genes cancer 3: 3-15, 2012

23. Babashah S, Sadeghizadeh M, Hajifathali A, et al. Targeting of the signal transducer Smo links microRNA-326 to the oncogenic Hedgehog pathway in CD34+ CML stem/progenitor cells. Int J Cancer 133: 579-589, 2013.

24. White SL, Belov L, Barber N, et al. Immunophenotypic changes induced on human HL60 leukaemia cells by $1 \alpha$, 25-dihydroxyvitamin D 3 and 12-O-tetradecanoyl phorbol13-acetate. Leuk Res 29: 1141-1151, 2005.

25. Dalton WJ, Ahearn M, McCredie KB, et al. HL-60 cell line was derived from a patient with FAB-M2 and not FAB-M3. Blood 71: 242-247, 1988.

26. Burak UZ, Tatonyan SC, Sayitoglu M, et al. Renin-Angiotensin System (RAS) Expressions in Myeloid Leukemic Cell Lines. UHOD 4: 264-268, 2013.

27. Emamdoost F, Khanahmad H, Ganjalikhani-hakemi M, et al. The miR-125a-3p Inhibits TIM-3 Expression in AML Cell Line HL-60 In Vitro. Indian J Hematol Blood Transfus 33(3): 342347, 2016.
28. Ekimler S, Sahin K. Computational methods for microRNA target prediction. Genes 5: 671-683, 2014.

29. Kikushige Y, Shima T, Yuda J, et al. Leukemogenic Function of TIM-3, a Leukemia Stem Cell Marker, in Acute Myelogenous Leukemia and Myelodysplastic Syndromes. Blood 120: 2983-2983, 2012.

30. Zhu C, Anderson AC, Schubart A, et al. The Tim-3 ligand galectin-9 negatively regulates $T$ helper type 1 immunity. Nat Immunol 6: 1245-1252, 2005.

31. Testa U, Riccioni R. Deregulation of apoptosis in acute myeloid leukemia. Haematologica 92: 81-94, 2007.

32. Konopleva M, Tari AM, Estrov Z, et al. Liposomal Bcl-2 antisense oligonucleotides enhance proliferation, sensitize acute myeloid leukemia to cytosine-arabinoside, and induce apoptosis independent of other antiapoptotic proteins. Blood 95(: 3929-3938, 2000

33. Lee J, Kotliarova S, Kotliarov Y, et al. Tumor stem cells derived from glioblastomas cultured in bFGF and EGF more closely mirror the phenotype and genotype of primary tumors than do serum-cultured cell lines. Cancer Cell 9: 391-403, 2006.

\section{Correspondence:}

Dr. Mazdak GANJALIKHANI-HAKEMI

Isfahan University of Medical Sciences

School of Medicine

Department of Immunology

ISFAHAN / IRAN

Tel: +98-31-37929082

email: mghakemi@med.mui.ac.ir 